\title{
FATORES DE RISCO ASSOCIADOS À MORTALIDADE INFANTIL EM DUAS ÁREAS DA REGIÃO METROPOLITANA DE SÃO PAULO (BRASIL), 1984-1985. PROPOSTA DE INSTRUMENTOS PREDITIVOS*
}

\author{
Chester L. G. Cesar**
}

\begin{abstract}
CESAR, C. L. G. Fatores de risco associados à mortalidade infantil em duas áreas da região metropolitana de São Paulo (Brasil), 1984-1985. Proposta de instrumentos preditivos. Rev. Saúde públ., S. Paulo, 24: 300-10, 1990.
\end{abstract}

RESUMO: Estudou-se a aplicação do conceito de "risco" na área materno-infantil, partindo da proposta da Organização Mundial de Saúde relativa ao "enfoque de risco" na organização dos serviços de saúde. O estudo concentrou-se no desenvolvimento de instrumentos de identificação de grupos de alto risco de óbito infantil, seja no período neonatal, seja no período pós-neonatal, e baseou-se em estudo de casocontrole. O grupo de casos correspondeu a óbitos registrados de menores de um ano de idade, ocorridos nos anos de 1984 e 1985, de pais residentes nos municípios de Cotia e Vargem Grande Paulista, SP (Brasil), totalizando 149 óbitos (casos). O grupo controle foi formado por uma amostra probabilística de 216 crianças nascidas em 1984, filhas de pais residentes nos munićpios estudados e que sobreviveram ao primeiro ano de vida. As mães de ambos os grupos responderam a um questionário, aplicado em entrevistas domiciliárias, para a identificação de variáveis independentes associadas ao óbito infantil. As variáveis que mostraram associação estatisticamente significante foram agrupadas em quatro escalas de risco: para uso em pré-natal; para uso por ocasião do parto; para uso no período neonatal; e para uso em puericultura após o período neonatal. As variáveis participaram nas escalas ponderadas pelos valores das razões dos produtos cruzados. As escalas apresentaram diferentes pontos de corte e a cada um destes correspondeu uma dada sensibilidade, especificidade e poder preditivo.

DESCRITORES: Mortalidade infantil. Fatores de risco. Grupos de risco. Valor de predição de testes.

\section{1 - INTRODUÇÃO}

A mortalidade na infância é talvez uma das áreas da saúde que sofreu maiớ impacto nos últimos cem anos. McKeown e Lowe ${ }^{6}$, analisando a tendência desta mortalidade na Inglaterra e Gales, a partir de meados do século passado, mostra queda acentuada e constante a partir de 1900. Esta tendência deve refletir, de certo modo, o que ocorreu com os atuais países industrializados, principalmente os europeus, que tivera uma história de urbanização, industrialização e transformaçōes econômicas, políticas e sociais muito semelhante.

Mais recentemente, Rico-Velasco ${ }^{10}$, analisando a tendência da mortalidade infantil no período de 1950-55 a 1975-80, mostra que em nível mundial houve uma queda acentuada. Para os países desenvolvidos, como um todo, esta queda foi de aproximadamente $66 \%$, passando de 56 para 19 6́bitos por 1.000 nascidos vivos. Para o grupo de países subdesenvolvidos esta queda foi de 164 para aproximadamente 100 óbitos por 1.000 nascidos vivos, representando uma queda relativa de $40 \%$.

Além dos grandes diferenciais de saúde entre os vários países, sabe-se que os valores dos coeficientes analisados são valores médios e que, freqüentemente, encobrem grandes diferenças entre subgrupos populacionais $2,4,9$. Isto decorre não somente das condiçōes diversas de vida dos diferentes grupos sociais, mas também de uma diferença marcante na utilização de serviços de saúde por estes grupos sociais, com uma sub-utilização pelos grupos mais desfavorecidos, do ponto de vista socio-econômico ${ }^{2,3,5,7}$.

Este conhecimento epidemiologico acumulado vem sendo progressivamente agregado às políticas e programas de saúde. Mais recentemente, há tendếncia na área materno-infantil de incorporá-lo pela operacionalização do conceito de risco que permite identificar grupos de alto risco. Vários trabalhos foram publicados neste sentido. Em 1978, a Organizaçăo Mundial de Saúde (OMS) divulgou publicação8, onde a utilização do conceito de risco na área materno-infantil vem melhor sis-

\footnotetext{
* Trabalho subvencionado pelo Conselho Nacional de Desenvolvimento Científico e Tecnologico (CNPq - Processo $403657 / 84$.

Síntese da tese de doutorado apresentada ao Departamento de Epidemiologia da Faculdade de Saúde Pública da Universidade de São Paulo, em 1989, sob o mesmo título.

* Departamento de Epidemiologia da Faculdade de Saúde Pública da Universidade de São Paulo - Av. Dr. Arnaldo, 715 - 01255 - Sảo Paulo, SP - Brasil.
} 
tematizada e intimamente articulada com uma estratégia de reorganização de serviços. Posteriormente, dois novos textos sobre o assunto foram publicados 1,13 , representando, por um lado, uma evolução do texto original de 1978 e, por outro, uma revisão do material desenvolvido em vários seminários efetuados.

A idéia central da proposta do enfoque de risco na organização dos serviços de saúde decorre da constatação da distribuição desigual dos "danos" (efeito indesejável - doença, acidente ou morte) entre os diversos grupos populacionais. Esta citada distribuição significa que grupos populacionais têm diferentes probabilidades de apresentarem danos à saúde, isto é, têm diferentes riscos de adoecerem, sofrerem acidentes ou irem a óbito. Estas diferenças nos riscos decorrem do fato de que alguns indivíduos apresentam características próprias ou estão sujeitos a circunstâncias que fazem com que a probabilidade de ocorrência de um dano à sua saúde seja maior do que para outros indivíduos sem as mesmas características, ou não expostos às mesmas circunstâncias. Estas características ou circunstâncias são denominadas fatores de risco e pela sua própria definição são observáveis ou identificáveis antes do evento a que estão associados. Esta associação pode ser causal ou não, porém a presença do fator de risco indica probabilidade maior de aparecimento do dano à saúde em questão.

O fato de que a presença de fatores de risco possa ser observada anteriormente ao aparecimento do dano abre duas possibilidades do ponto de vista operacional. A primeira delas corresponde ao possível controle ou eliminação desses fatores, impedindo o aparecimento do dano, e a segunda possibilidade, possivelmente a mais importante, é que os fatores de risco permitem a identificação de grupos de alto risco que devam ser objeto de atenção especial por parte dos serviços de saúde, no sentido de compensar estes riscos através de ações de saúde.

A partir destas idéias básicas, a estratégia de risco propõe que se analisem os serviços de saúde frente às prioridades da sua área de atuação, e que eles redirecionem suas atividades para que, dispensando uma assistência básica a todos os indivíduos, concentrem esforços naqueles que pertençam aos grupos de alto risco. Ou seja, no dizer de Backett e col.1 - "algo para todos e mais para os que têm necessidade, em proporção a essa necessidade".

Esta estratégia de risco implica desenvolver instrumentos que permitam identificar os grupos de acordo com o grau de risco que apresentem. $O$ presente trabalho procura se ater mais ao desenvolvimento destes instrumentos para identificação de grupos de alto risco de óbito infantil, seja no período neonatal, seja no pós-neonatal.
Os autores da proposta de enfoque de risco frisam a importância de aplicar esta metodologia mais amplamente e em diferentes circunstâncias para que se possa melhor avaliá-la e aperfeiçoála. O presente trabalho deve ser visto sob esta perspectiva, sem ter nenhuma pretensão de esgotar as dificuldades existentes nesta metodologia. Pretende, também, estimular, nos serviços de saúde da área geográfica estudada, um maior debate sobre as questões relativas ao enfoque de risco na área materno-infantil.

\section{2 - METODOLOGIA}

A área onde se desenvolveu o presente trabalho compreende os municípios de Cotia e Vargem Grande Paulista, pertencentes à área metropolitana de São Paulo.

Para identificação dos fatores de risco (variáveis independentes) associados à mortalidade infantil (variável dependente) utilizou-se estudo tipo caso-controle. As variáveis independentes foram selecionadas a partir de dados da literatura e as informações foram obtidas através de entrevistas com as mães, realizadas por seis visitadoras domiciliárias ligadas aos serviços locais de saúde. Estas variáveis agrupam-se em quatro blocos: fatores maternos, fatores infantis, fatores ambientais (ambiente físico) e fatores sócioeconômico-culturais.

\section{1 - Determinação do tamanho da amostra}

A determinação do tamanho da amostra levou em conta, basicamente, a magnitude do valor da razão dos produtos cruzados das variáveis em estudo (RPC) e a prevalência destas variáveis na população (po).

Considerou-se que valores muito baixos da razão dos produtos cruzados ou fatores de risco de freqüência muito baixa na população não deveriam ser incluídos no estudo. Além disto, optou-se por um nível de significância de $5 \%($ alfa $=0,05)$ e um beta $=0,10$. Inicialmente, levando-se em conta a necessidade de um tamanho de amostra viável do ponto de vista operacional, definiram-se o valor mínimo do risco relativo em 2,5 e a prevalência mínima do fator em $10 \%(0,10)$. Para estes valores, - tamanho da amostra foi calculado através da fórmula descrita por Schlesselman ${ }^{11}$, conforme pode ser visto a seguir:

$$
\mathrm{n}=2 \overline{\mathrm{p}} \overline{\mathrm{q}}\left(\mathrm{z}_{\alpha}+\mathrm{z}_{\beta}\right)^{2} /\left(\mathrm{p}_{1}-\mathrm{p}_{0}\right)^{2}
$$

que no caso específico de um $\alpha=0,05$ (bicaudal) e um $\beta=0,10$, transforma-se em:

$$
\mathrm{n}=\frac{21 \overline{\mathrm{p}} \overline{\mathrm{q}}}{\left(\mathrm{p}_{1}-\mathrm{p}_{0}\right)^{2}}
$$


onde:

21 é o resultado de:

$$
2\left(z_{\alpha}+z_{\beta}\right)^{2}=2(1,96+1,28)^{2}
$$

que é igual a 20,9952 (aproximadamente 21) $\mathrm{p}_{\mathrm{o}}=$ prevalência mínima dos fatores de risco $=0,10$

$\mathrm{R}=$ valor mínimo da $\mathrm{RPC}=2,5$

$$
p_{1}=\frac{p_{0} R}{\left(1+p_{0}(R-1)\right.}=\frac{(0,10 \times 2,5)}{(1+0,10(2,5-1))}=0,22
$$

$$
\begin{aligned}
& \bar{p}=1 / 2\left(p_{1}+p_{0}\right)=1 / 2(0,22+0,10)=0,16 \\
& \bar{q}=1-p=1-0,16=0,84
\end{aligned}
$$

portanto:

$$
\mathrm{n}=\frac{(21 \times 0,16 \times 0,84)}{(0,22-0,10)^{2}}=\frac{2,8224}{0,0144}=196
$$

Assim, o tamanho da amostra estabelecido foi de aproximadamente 200 casos e 200 controles (sem inclusão das possíveis perdas). Considerou-se ainda que valores um pouco menores do risco relativo para fatores com prevalência acima de $10 \%$, bem como prevalências um pouco menores do que a definida, para valores do risco relativo acima de 2,5 , estariam incluídos neste tamanho de amostra. Desta maneira estar-se-iam privilegiando os fatores de risco fortemente associados à mortalidade infantil (alto valor do risco relativo), ainda que de baixa prevalência na população e os fatores de risco que, embora não apresentem uma associação tão forte com a mortalidade infantil (valor do risco relativo menor), sejam de alta prevalência na população.

Deve ser lembrado, ainda, que o valor estabelecido de $B(0,10)$ altera-se com a diminuição do número de casos analisados quando estratificam-se os óbitos em neonatais e infantis tardios, na construção das escalas.

\section{2 - População em estudo}

\subsection{1 - Grupo dos casos (obitos)}

Levando-se em conta a necessidade calculada de 200 óbitos e 200 controles, optou-se por trabalhar com o universo de bbitos infantis correspondentes aos anos de 1984 e 1985. Definiu-se, assim, o grupo caso como o total de óbitos registrados de crianças menores de um ano, ocorridos em 1984 e 1985, filhos de pais residentes em Cotia e Vargem Grande Paulista.
Dado o fato de alguns destes óbitos ocorrerem e serem registrados fora desta área, houve necessidade de identificarem-se os locais onde estes acontecem e os cartórios onde são registrados. Foram utilizadas as listagens da Companhia de Processamento de Dados do Estado de São Paulo (PRODESP), de 1982, a partir das quais foram relacionados os cartórios onde os óbitos de residentes em Cotia e Vargem Grande Paulista foram registrados.

Para a identificação dos óbitos ocorridos em 1984 e 1985, coletou-se na Fundação Sistema Estadual de Análise de Dados (FSEADE) o conjunto de todos os atestados de 6 bito, referentes a estes dois anos, nos cartórios listados. Levantou-se também o total de informações dos óbitos de menores de um ano nos registros dos cemitérios de Cotia e Vargem Grande Paulista. Os atestados de óbitos, assim identificados, foram o ponto de referência para a localização da residência das famílias (mães) do grupo de casos.

O grupo original dos casos corresponde ao total de óbitos registrados de menores de um ano ocorridos em 1984 e 1985, sendo igual a $179(94+85)$ óbitos; deste total, foram entrevistadas 149 mães (perda de $16,7 \%$ ).

\subsection{2 - Grupo dos controles (sobreviventes)}

O grupo controle é formado por crianças com mais de doze meses, nascidas no ano de 1984, registradas em cartório e filhas de pais residentes nos municípios de Cotia e Vargem Grande Paulista.

A sistemática de identificação do grupo controle partiu também de uma listagem da PRODESP indicando os cartórios de registro dos nascimentos de crianças de pais residentes em Cotia e Vargem Grande Paulista ocorridos em 1983 e registrados no mesmo ano ou até 31 de março de 1984. Foram incluídos na listagem os cartórios de nascimentos de crianças de pais residentes na área em estudo que apresentaram em 1983 cinco ou mais registros.

A seguir, foram revistos todos os mapas de nascimento dos Cartórios listados, anotando-se para cada cartório número e data de registro, data de nascimento, sexo da criança e o município de residência dos pais (Cotia ou Vargem Grande Paulista). Estes dados formaram uma listagem do universo de nascidos vivos, em 1984, de pais residentes na área de estudo, e dela foi retirada uma amostra de 284 crianças que correspondeu ao grupo controle.

Para cada controle incluído na amostra foram levantados no cartório correspondente, após autorização judicial, o nome da criança e o nome e endereço dos pais, informaçðes estas que permitiram a entrevista domiciliária dos controles. 
Das 284 sorteadas, foram excluídas como não pertencentes ao grupo controle 14 crianças (três não residentes na área, um registro cancelado, uma criança nascida em 1983 e nove que foram a 6 bito, sendo que destas, sete estão incluídas no grupo de obitos e duas săo perdas). Das 270 crianças do grupo controle, 216 foram efetivamente entrevistadas (perda de 20,4\%).

\subsection{3 - Amostra dos nascidos vivos em 1984}

Além dos dois grupos fundamentais estudados, isto é, o grupo de obitos (casos) e o grupo de sobreviventes (controles), foi também possivel estudar um terceiro grupo que correspondeu a uma amostra probabilística dos nascidos vivos em 1984, em Cotia e Vargem Grande Paulista. Este grupo vem a ser o proprio grupo de nascidos vivos, originalmente sorteados para a seleção dos controles. Esta amostra foi de tamanho 223 (216 sobreviventes e 7 óbitos).

\section{3 - Processamento e análise de dados}

Foi utilizado o pacote estatístico SPSS (Statistical Package for the Social Sciences) para testes de consistência e para a obtenção de tabelas de freqüência e testes de associação. $O$ cálculo da RPC foi feito diretamente das tabelas de contingência.

A partir dos valores das RPCs foram elaboradas escalas de predição para grupos de risco, calculando-se, para diferentes pontos de cortes, a sensibilidade, a especificidade, os números de verdadeiros positivos, verdadeiros negativos, falsos positivos, falsos negativos, bem como o poder preditivo positivo e negativo das escalas.

\section{3- ANÁLISE DA ASSOCIAÇÃO ENTRE OS DIFERENTES FATORES DE RISCO E A MORTALIDADE NO PRIMEIRO ANO DE VIDA}

\section{1 - Categorização das variáveis}

A análise do comportamento das variáveis independentes (fatores de risco) foi o ponto de partida para a categorização das mesmas, em dois grupos: 0 de risco e 0 de não risco. Esta categorização consistiu em dicotomizar estas variáveis, sendo que no caso das qualitativas, as possíveis alternativas de categorias assumidas foram alocadas nos grupos de risco ou de não risco. No caso das variáveis quantitativas, os possíveis valores assumidos foram também classíficados como de risco ou não risco. Neste último caso, freqüuentemente a categorizaçăo consistiu em estabelecer um ponto de corte, tanto para as variáveis contínuas como para as discretas.

Este processo de categorizaçăo levou em conta, além do comportamento destas variáveis no presente estudo, as informaçð̄es já existentes na literatura.
Algumas poucas variáveis não foram estudadas separadamente e sim associadas a uma ou mais, criando-se, desta forma, novas variáveis.

\section{2 - Análise da associação estatística}

Trinta e quatro variáveis independentes foram analisadas quanto à sua associação estatística com a variável dependente, utilizando a estatística "Qui-Quadrado". Na verdade, esta associação foi analisada separadamente para mortalidade neonatal e para a pós-neonatal, resultando, assim, 68 tabelas de contingência, para as quais foi calculado o Qui-Quadrado. Em quinze delas houve associação estatisticamente significante, em nível de $5 \%$. Para todas as variáveis com associação foi calculada a RPC.

As variáveis independentes (fatores de risco) que mostraram associação estatística com um dos grupos de 6bitos ou com ambos foram: altura da mãe, número de gestaçð̃es anteriores, intervalo entre o nascimento da criança em estudo e o nascimento anterior, intervalo interpartal médio, nova gestação após o nascimento da criança em estudo, história prévia de perda fetal, história prévia de óbito de menor de um ano, cobertura da mãe por serviços de saúde, acompanhamento do pré-natal, sexo da criança, peso ao nascer, doença crônica da criança, migração recente, tempo morando com o atual companheiro e tipo de habitação.

\section{3 - Elaboração das escalas de risco}

As variáveis independentes que mostraram associação estatística com a mortalidade infantil foram agrupadas em quatro escalas de risco: para uso em pré-natal; para uso por ocasião do parto; para uso no período neonatal; para uso em puericultura, após o período neonatal.

A primeira escala, a ser usada em pré-natal, é composta por variáveis possíveis de serem identificadas numa primeira entrevista com a mãe e visa a identificar os grupos cujas características indiquem maior risco de óbito de criança no primeiro ano de vida (neonatal ou infantil tardio). Entram na composição desta escala as variáveis associadas à mortalidade neonatal e/ou à mortalidade infantil tardia.

A segunda escala tem por finalidade identificar grupos de alto risco que devam ser objeto de assistência especial por ocasião do parto, visando a reduzir a mortalidade neonatal. Esta escala é elaborada a partir das variáveis associadas à mortalidade neonatal, passiveis de serem identificadas no período imediatamente anterior ao parto.

A terceira, da mesma forma que a anterior, visa a reduzir a mortalidade neonatal e deve identificar as crianças que necessitam de uma assistência especial durante o período neonatal. A elaboração 


\section{TABELA 1}

Escala 1 - Escala para identificar, durante o período pré-natal, grupos de risco para óbito infantil. Cotia e Vargem Grande Paulista, 1984-1985.

\begin{tabular}{|c|c|c|}
\hline Variáveis & Valores & $\mathrm{RPC}^{*}$ \\
\hline Altura das mães & $\begin{array}{l}\text { abaixo de } 150 \mathrm{~cm} \\
150 \mathrm{~cm} \text { ou mais }\end{array}$ & $\begin{array}{l}2,15 \\
1,00\end{array}$ \\
\hline Número prévio de gestações & $\begin{array}{l}4 \text { ou mais } \\
\text { Até } 3\end{array}$ & $\begin{array}{l}2,15 \\
1,00\end{array}$ \\
\hline $\begin{array}{l}\text { Intervalo entre a data } \\
\text { provável do parto e o parto } \\
\text { anterior }\end{array}$ & $\begin{array}{l}\text { Até } 18 \text { meses } \\
18 \text { meses ou mais }\end{array}$ & $\begin{array}{l}2,29 \\
1,00\end{array}$ \\
\hline Intervalo interpartal médio & $\begin{array}{l}\text { Até } 24 \text { meses } \\
24 \text { meses ou mais }\end{array}$ & $\begin{array}{l}3,20 \\
1,00\end{array}$ \\
\hline $\begin{array}{l}\text { História prévia de perda } \\
\text { fetal }\end{array}$ & $\begin{array}{l}\text { Sim } \\
\text { Não }\end{array}$ & $\begin{array}{l}2.83 \\
1,00\end{array}$ \\
\hline $\begin{array}{l}\text { História prévia de óbito de } \\
\text { criança menor de } 1 \text { ano }\end{array}$ & $\begin{array}{l}\text { Sim } \\
\text { Não }\end{array}$ & $\begin{array}{l}3,38 \\
1,00\end{array}$ \\
\hline $\begin{array}{l}\text { Cobertura das mães por ser- } \\
\text { viços de saúde }\end{array}$ & $\begin{array}{l}\text { Nāo } \\
\text { Sim }\end{array}$ & $\begin{array}{l}1,96 \\
1,00\end{array}$ \\
\hline Migração recente & $\begin{array}{l}\text { Sim } \\
\text { Não }\end{array}$ & $\begin{array}{l}5,28 \\
1,00\end{array}$ \\
\hline $\begin{array}{l}\text { Tempo morando com o com- } \\
\text { panheiro }\end{array}$ & $\begin{array}{l}\text { Menos de } 24 \text { meses } \\
24 \text { meses ou mais }\end{array}$ & $\begin{array}{l}2,26 \\
1,00\end{array}$ \\
\hline Tipo de habitação & $\begin{array}{l}\text { Outros } \\
\text { Casa ou apto. }\end{array}$ & $\begin{array}{l}5,83 \\
1,00\end{array}$ \\
\hline
\end{tabular}

* RPC = Razão dos Produtos Cruzados

desta escala é feita a partir das variáveis associadas à mortalidade neonatal, identificáveis antes do parto, e das demais variáveis associadas a esta mortalidade e somente identificáveis após o parto.

A quarta escala deve identificar as crianças que apresentam um risco maior de óbito no periodo pós-neonatal. Ela é composta pelas variáveis que apresentaram associação com a mortalidade infantil tardia.

As escalas foram elaboradas a partir dos valores da RPC. Algumas das variáveis que entraram na composição das escalas mostraram associação estatística, tanto com a mortalidade neonatal como com a infantil tardia, apresentando valores diferentes da RPC. Nestes casos entrou na composição das escalas o maior valor da RPC. Isto ocorreu para as variáveis: pré-natal, peso ao nascer e tipo de habitação.

As Tabelas 1 a 4 mostram as variáveis que compóem as quatro escalas com os respectivos va-
TABELA 2

Escala 2 - Escala para identificar, no período imediatamente anterior ao parto, grupos de risco para óbito neonatal. Cotia e Vargem Grande Paulista, 1984-1985.

\begin{tabular}{lll}
\hline \multicolumn{1}{c}{ Variáveis } & \multicolumn{2}{c}{ Valores da RPC } \\
\hline Altura das mães & $\begin{array}{l}\text { abaixo de } 150 \mathrm{~cm} \\
150 \mathrm{~cm} \text { ou mais }\end{array}$ & 2,15 \\
& 1,00 \\
Número prévio de gestaçōes & 4 ou mais & 2,15 \\
& Até 3 & 1,00 \\
Intervalo entre o nascimento & Até 18 meses & 2,17 \\
e o parto anterior & 18 meses ou mais & 1,00 \\
& & \\
História prévia de perda & Sim & 2,38 \\
fetal & Não & 1,00 \\
História prévia de óbito de & Sim & 3,83 \\
criança menor de 1 ano & Não & 1,00 \\
Cobertura das mães por ser- - Não & Sim & 1,96 \\
viços de saúde & Não & 1,00 \\
Pré-natal & Sim & 4,19 \\
& Sim & 1,00 \\
Migração recente & Não & 5,28 \\
& & 1,00 \\
Tempo morando com o com- & Menos de 24 meses & 2,26 \\
panheiro & 24 meses ou mais & 1,00 \\
Tipo de habitação & Outros & 4,30 \\
& Casa ou apto. & 1,00 \\
\hline
\end{tabular}

* RPC = Razão dos Produtos Cruzados

lores da RPC. Na Tabela 1, que corresponde à escala para uso em pré-natal, encontram-se listadas as 10 variáveis que a compõem, estando cada uma delas dicotomizada em risco e não risco, correspondendo ao grupo risco o respectivo valor da RPC e ao grupo não risco o valor 1 . A primeira variável que compõe a escala é a altura da mãe e ela foi dicotomizada em altura inferior a $150 \mathrm{~cm}$ e altura igual ou superior a $150 \mathrm{~cm}$ (Tabela 1). O primeiro grupo corresponde ao de risco e foi ponderado em 2,15, valor da RPC. Se o valor da altura da mãe foi superior a $150 \mathrm{~cm}$, isto é, se ela pertence ao grupo não risco, o valor da RPC computado foi 1.

O mesmo procedimento foi feito para as variáveis seguintes, computando-se para cada uma delas o respectivo valor da RPC quando se tratava de grupo de risco ou o valor 1 , quando pertencente ao grupo de não risco.

Para compor o total de pontos para cada indivíduo, os diferentes valores encontrados foram então multiplicados. Considere-se, por exemplo, uma mãe que pertença aos grupos de risco para as 


\section{TABELA 3}

Escala 3 - Escala para identificar, ao nascimento, grupos de risco de b́bito neonatal. Cotia e Vargem Grande Paulista, 1984-1985.

\begin{tabular}{|c|c|}
\hline Variáveis & Valores \\
\hline Altura das mães & $\begin{array}{l}\text { abaixo de } 150 \mathrm{~cm} \\
150 \mathrm{~cm} \text { ou mais }\end{array}$ \\
\hline Número prévio de gestações & $\begin{array}{l}4 \text { ou mais } \\
\text { Até } 3\end{array}$ \\
\hline $\begin{array}{l}\text { Intervalo entre o nascimento } \\
\text { e o parto anterior }\end{array}$ & $\begin{array}{l}\text { Até } 18 \text { meses } \\
18 \text { meses ou mais }\end{array}$ \\
\hline $\begin{array}{l}\text { História prévia de perda } \\
\text { fetal }\end{array}$ & $\begin{array}{l}\text { Sim } \\
\text { Não }\end{array}$ \\
\hline $\begin{array}{l}\text { História prévia de óbito de } \\
\text { criança menor de } 1 \text { ano }\end{array}$ & $\begin{array}{l}\text { Sim } \\
\text { Não }\end{array}$ \\
\hline $\begin{array}{l}\text { Cobertura das mães por ser- } \\
\text { viços de saúde }\end{array}$ & $\begin{array}{l}\text { Näo } \\
\text { Sim }\end{array}$ \\
\hline Pré-natal & $\begin{array}{l}\text { Não } \\
\text { Sim }\end{array}$ \\
\hline Sexo da criança & $\begin{array}{l}\text { Masculino } \\
\text { Feminino }\end{array}$ \\
\hline Peso ao nascer & $\begin{array}{l}\text { Até } 2.500 \mathrm{gr} \\
2.500 \mathrm{gr} \text { ou mais }\end{array}$ \\
\hline Migração recente & $\begin{array}{l}\text { Sim } \\
\text { Não }\end{array}$ \\
\hline $\begin{array}{l}\text { Tempo morando com o com- } \\
\text { panheiro }\end{array}$ & $\begin{array}{l}\text { Menos de } 24 \text { meses } \\
24 \text { meses ou mais }\end{array}$ \\
\hline Tipo de habitação & $\begin{array}{l}\text { Outros } \\
\text { Casa ou apto. }\end{array}$ \\
\hline
\end{tabular}

* RPC = Razão dos Produtos Cruzados

variáveis altura, história prévia de óbito fetal e migração recente e que para as demais variáveis situe-se nos grupos de não risco. Neste caso ela teria para a variável altura o valor da RPC de 2,15 , para a história prévia de perda fetal, de 2,38, e para a migração recente, de 5,28. Para as demais variáveis, o valor da RPC seria igual a 1 . Esta mãe teria então o valor final dos seus pontos na escala de risco igual ao produto destes três valores diferentes de 1 , ou seja, $27(2,15 \times 2,38 \times 5,28)$.

As possibilidades de combinaçōes dos valores destas 10 variáveis da escala de pré-natal são inúmeras, variando de um valor mínimo igual a 1, ou seja, o correspondente aos indivíduos que se situaram no grupo "não risco" para todas elas até um máximo correspondente às mães que se tivessem situado no grupo de risco para todas as 10 variáveis (valor aproximadamente igual a 42.102).

A definição da pontuação das quatro escalas foi
TABELA 4

Escala 4 - Escala para identificar, após o nascimento, grupos de risco para óbito neonatal. Cotia e Vargem Grande Paulista, 1984-1985.

\begin{tabular}{llr}
\hline \multicolumn{1}{c}{ Variáveis } & \multicolumn{2}{c}{ Valores da $\mathrm{RPC}^{*}$} \\
\hline Intervalo entre o nascimento & Até 18 meses & 2,29 \\
e o parto anterior & 18 meses ou mais & 1,00 \\
& & 3,20 \\
Intervalo interpartal médio & Até 24 meses & 1,00 \\
& 24 meses ou mais & 5,84 \\
Pré-natal & Năo & 1,00 \\
& Sim & 3,49 \\
Peso ao nascer & Até 2.500 gr & 1,00 \\
& 2.500 gr ou mais & 4,46 \\
Doença crônica da criança & Sim & 1,00 \\
& Não & 5,83 \\
Tipo de habitaçăo & Outros & 1,00 \\
& Casa ou apto. & \\
\hline
\end{tabular}

* RPC = Razão dos Produtos Cruzados

TABELA 5

Distribuição dos intervalos dos valores das razões dos produtos cruzados (RPC) segundo os graus das escalas. Cotia e Vargem Grande Paulista, 1984-1985.

\begin{tabular}{ccc}
\hline $\begin{array}{c}\text { Graus } \\
\text { das } \\
\text { Escalas }\end{array}$ & \multicolumn{2}{c}{ Intervalos de valores das RPC $^{*}$} \\
\cline { 2 - 3 } & ESCALAS 1, 2 e 3(1) & ESCALA 4 (2) \\
\hline $8+$ & $60,0 \mathrm{e}+$ & $50,0 \mathrm{e}+$ \\
7 & $25,0 \vdash 60,0$ & $30,0 \vdash 50,0$ \\
6 & $15,0 \vdash 25,0$ & $20,0 \vdash 30,0$ \\
5 & $10,0 \vdash 15,0$ & $10,0 \vdash 20,0$ \\
4 & $5,0 \vdash 10,0$ & $6,0 \vdash 10,0$ \\
3 & $2,5 \vdash 5,0$ & $3,5 \vdash 6,0$ \\
2 & $2,0 \vdash 2,5$ & $3,0 \vdash 3,5$ \\
1 & $1,1 \vdash 2,0$ & $2,0 \vdash 3,0$ \\
0 & $<1,1$ & $<2,0$ \\
\end{tabular}

(1) Escala 1 - Pré-Natal,

Escala 2 - Parto,

Escala 3 - Período neonatal,

(2) Escala 4 - Período pós-natal.

* RPC - Razão dos Produtos Cruzados.

feita a partir da distribuição dos grupos estudados, o de óbitos e o de sobreviventes, procurando-se possibilitar alternativas de escolha para o ponto de corte mais adequado às condições reais de recursos humanos e materiais, por um lado, e de maior ou menor sensibilidade e especificidade de outro, 


\section{TABELA 6}

Classificação dos grupos de óbitos, sobreviventes e nascidos vivos pela Escala 1 (Pré-natal).

Cotia e Vargem Grande Paulista, 1984-1985.

\begin{tabular}{|c|c|c|c|c|c|c|c|c|c|c|c|c|}
\hline \multirow{2}{*}{$\begin{array}{c}\text { Pontuação } \\
\text { da } \\
\text { escala }\end{array}$} & \multicolumn{4}{|c|}{ Óbitos } & \multicolumn{4}{|c|}{ Sobreviventes } & \multicolumn{4}{|c|}{ Amostra de Nascidos Vivos em 1984} \\
\hline & $N^{2}$ & $\%$ & $N^{\circ}$ acum & $\%$ acum & $\mathbf{N}^{2}$ & $\%$ & $N^{2}$ acum & $\%$ acum & $\mathrm{N}^{2}$ & $\%$ & $N^{\circ}$ acum & $\%$ acum \\
\hline $\begin{array}{l}8 e+ \\
7 \\
6 \\
5 \\
4 \\
3 \\
2 \\
1 \\
0\end{array}$ & $\begin{array}{c}22 \\
13 \\
11 \\
17 \\
20 \\
11 \\
28 \\
6 \\
21\end{array}$ & $\begin{array}{r}14,8 \\
8,7 \\
7,4 \\
11,4 \\
13,4 \\
7,4 \\
18,8 \\
4,0 \\
14,1\end{array}$ & $\begin{array}{c}22 \\
35 \\
46 \\
63 \\
83 \\
94 \\
122 \\
128 \\
149\end{array}$ & $\begin{array}{c}14,8 \\
23,5 \\
30,9 \\
42,3 \\
55,7 \\
63,1 \\
81,9 \\
85,9 \\
100,0\end{array}$ & $\begin{array}{c}6 \\
4 \\
4 \\
7 \\
28 \\
27 \\
56 \\
14 \\
70\end{array}$ & $\begin{array}{r}2,8 \\
1,9 \\
1,9 \\
3,2 \\
13,0 \\
12,5 \\
25,9 \\
6,5 \\
32,4\end{array}$ & $\begin{array}{c}6 \\
10 \\
14 \\
21 \\
49 \\
76 \\
132 \\
146 \\
216\end{array}$ & $\begin{array}{r}2,8 \\
4,6 \\
6,5 \\
9,7 \\
22,7 \\
35,2 \\
61,1 \\
67,6 \\
100,0\end{array}$ & $\begin{array}{c}7 \\
5 \\
5 \\
7 \\
30 \\
28 \\
57 \\
14 \\
70\end{array}$ & $\begin{array}{r}3,1 \\
2,2 \\
2,2 \\
3,1 \\
13,5 \\
12,6 \\
25,6 \\
6,3 \\
31,4\end{array}$ & $\begin{array}{c}7 \\
12 \\
17 \\
24 \\
54 \\
82 \\
139 \\
153 \\
223\end{array}$ & $\begin{array}{r}3,1 \\
5,4 \\
7,6 \\
10,8 \\
24,2 \\
36,8 \\
62,3 \\
68,6 \\
100,0\end{array}$ \\
\hline Total & 149 & 100,0 & & & 216 & 100,0 & & & 223 & 100,0 & & \\
\hline
\end{tabular}

\section{TABELA 7}

Classificação dos grupos de óbitos, sobreviventes e nascidos vivos pela Escala 2 (Parto).

Cotia e Vargem Grande Paulista, 1984-1985.

\begin{tabular}{|c|c|c|c|c|c|c|c|c|c|c|c|c|}
\hline \multirow{2}{*}{$\begin{array}{c}\text { Pontuação } \\
\text { da } \\
\text { escala }\end{array}$} & \multicolumn{4}{|c|}{ Óbitos } & \multicolumn{4}{|c|}{ Sobreviventes } & \multicolumn{4}{|c|}{ Amostra de N.V. em 1984} \\
\hline & $N^{2}$ & $\%$ & $\mathrm{~N}^{2}$ acum & $\%$ acum & $\mathbf{N}^{2}$ & $\%$ & $\mathrm{~N}^{2}$ acum & $\%$ acum & $\mathrm{N}^{2}$ & $\%$ & $\mathrm{~N}^{2}$ acum & $\%$ acum \\
\hline $\begin{array}{l}8 \mathrm{e}+ \\
7 \\
6 \\
5 \\
4 \\
3 \\
2 \\
1 \\
0\end{array}$ & $\begin{array}{c}11 \\
10 \\
8 \\
8 \\
12 \\
8 \\
14 \\
2 \\
8\end{array}$ & $\begin{array}{r}13,6 \\
12,3 \\
9,9 \\
9,9 \\
14,8 \\
9,9 \\
17,3 \\
2,5 \\
9,9\end{array}$ & $\begin{array}{l}11 \\
21 \\
29 \\
37 \\
49 \\
57 \\
71 \\
73 \\
81\end{array}$ & $\begin{array}{r}13,6 \\
25,9 \\
35,8 \\
45,7 \\
60,5 \\
70,4 \\
87,7 \\
90,1 \\
100,0\end{array}$ & $\begin{array}{c}5 \\
5 \\
4 \\
5 \\
23 \\
27 \\
60 \\
13 \\
74\end{array}$ & $\begin{array}{r}2,3 \\
2,3 \\
1,9 \\
2,3 \\
10,6 \\
12,5 \\
27,8 \\
6,0 \\
34,3\end{array}$ & $\begin{array}{c}5 \\
10 \\
14 \\
19 \\
42 \\
69 \\
129 \\
142 \\
216\end{array}$ & $\begin{array}{r}2,3 \\
4,6 \\
6,5 \\
8,8 \\
19,4 \\
31,9 \\
59,7 \\
65,7 \\
100,0\end{array}$ & $\begin{array}{c}6 \\
7 \\
6 \\
5 \\
24 \\
27 \\
61 \\
13 \\
74\end{array}$ & $\begin{array}{r}2,7 \\
3,1 \\
2,7 \\
2,2 \\
10,8 \\
12,1 \\
27,4 \\
5,8 \\
33,2\end{array}$ & $\begin{array}{c}6 \\
13 \\
19 \\
24 \\
48 \\
75 \\
136 \\
149 \\
223\end{array}$ & $\begin{array}{r}2,7 \\
5,8 \\
8,5 \\
10,8 \\
21,5 \\
33,6 \\
61,0 \\
66,8 \\
100,0\end{array}$ \\
\hline Total & 81 & 100,0 & & & 216 & 100,0 & & & 223 & 100,0 & & \\
\hline
\end{tabular}

\section{TABELA 8}

Classificação dos grupos de óbitos, sobreviventes e nascidos vivos pela Escala 3 (Neonatal).

Cotia e Vargem Grande Paulista, 1984-1985.

\begin{tabular}{|c|c|c|c|c|c|c|c|c|c|c|c|c|}
\hline \multirow{2}{*}{$\begin{array}{c}\text { Pontuação } \\
\text { da } \\
\text { escala }\end{array}$} & \multicolumn{4}{|c|}{ Óbitos } & \multicolumn{4}{|c|}{ Sobreviventes } & \multicolumn{4}{|c|}{ Amostra de N.V. em 1984} \\
\hline & $N^{2}$ & $\%$ & $N^{2}$ acum & $\%$ acum & $\mathrm{N}^{2}$ & $\%$ & $N^{2}$ acum & $\%$ acum & $N^{2}$ & $\%$ & $\mathrm{~N}^{2}$ acum & $\%$ acum \\
\hline $\begin{array}{l}8 \mathrm{e}+ \\
7 \\
6 \\
5 \\
4 \\
3 \\
2 \\
1 \\
0\end{array}$ & $\begin{array}{c}37 \\
14 \\
8 \\
1 \\
5 \\
8 \\
3 \\
4 \\
1\end{array}$ & $\begin{array}{r}45,7 \\
17,3 \\
9,9 \\
1,2 \\
6,2 \\
9,9 \\
3,7 \\
4,9 \\
1,2\end{array}$ & $\begin{array}{l}37 \\
51 \\
59 \\
60 \\
65 \\
73 \\
76 \\
80 \\
81\end{array}$ & $\begin{array}{r}45,7 \\
63,0 \\
72,8 \\
74,1 \\
80,2 \\
90,1 \\
93,8 \\
98,8 \\
100,0\end{array}$ & $\begin{array}{c}11 \\
11 \\
18 \\
5 \\
32 \\
41 \\
28 \\
39 \\
31\end{array}$ & $\begin{array}{r}5,1 \\
5,1 \\
8,3 \\
2,3 \\
14,8 \\
19,0 \\
13,0 \\
18,1 \\
14,4\end{array}$ & $\begin{array}{c}11 \\
22 \\
40 \\
45 \\
77 \\
118 \\
146 \\
185 \\
216\end{array}$ & $\begin{array}{r}5,1 \\
10,2 \\
18,5 \\
20,8 \\
35,6 \\
54,6 \\
67,6 \\
85,6 \\
100,0\end{array}$ & $\begin{array}{c}16 \\
13 \\
18 \\
5 \\
32 \\
41 \\
28 \\
39 \\
31\end{array}$ & $\begin{array}{r}7,2 \\
5,8 \\
8,1 \\
2,2 \\
14,3 \\
18,4 \\
12,6 \\
17,5 \\
13,9\end{array}$ & $\begin{array}{c}16 \\
29 \\
47 \\
52 \\
84 \\
125 \\
153 \\
192 \\
223\end{array}$ & $\begin{array}{r}7,2 \\
13,0 \\
21,1 \\
23,3 \\
37,7 \\
56,1 \\
68,6 \\
86,1 \\
100,0\end{array}$ \\
\hline Total & 81 & 100,0 & & & 216 & 100,0 & & & 223 & 100,0 & & \\
\hline
\end{tabular}




\section{TABELA 9}

Classificação dos grupos de óbitos, sobreviventes e nascidos vivos pela Escala 4 (Pós-neonatal).

Cotia e Vargem Grande Paulista, 1984-1985.

\begin{tabular}{|c|c|c|c|c|c|c|c|c|c|c|c|c|}
\hline \multirow{2}{*}{$\begin{array}{c}\text { Pontuação } \\
\text { da } \\
\text { escala }\end{array}$} & \multicolumn{4}{|c|}{ Óbitos } & \multicolumn{4}{|c|}{ Sobreviventes } & \multicolumn{4}{|c|}{ Amostra de N.V. em 1984} \\
\hline & $\mathbf{N}^{2}$ & $\%$ & No acum & $\%$ acum & $\mathrm{N}^{\mathbf{2}}$ & $\%$ & NNo acum & $\%$ acum & $N^{\circ}$ & $\%$ & No acum & $\%$ acum \\
\hline $\begin{array}{l}8 \mathrm{e}+ \\
7 \\
6 \\
5 \\
4 \\
3 \\
2 \\
1 \\
0\end{array}$ & $\begin{array}{c}15 \\
5 \\
11 \\
3 \\
3 \\
8 \\
6 \\
2 \\
15\end{array}$ & $\begin{array}{r}22,1 \\
7,4 \\
16,2 \\
4,4 \\
4,4 \\
11,8 \\
8,8 \\
2,9 \\
22,1\end{array}$ & $\begin{array}{l}15 \\
20 \\
31 \\
34 \\
37 \\
45 \\
51 \\
53 \\
68\end{array}$ & $\begin{array}{c}22,1 \\
29,4 \\
45,6 \\
50,0 \\
54,4 \\
66,2 \\
75,0 \\
77,9 \\
100,0\end{array}$ & $\begin{array}{r}5 \\
1 \\
11 \\
6 \\
9 \\
13 \\
31 \\
12 \\
128\end{array}$ & $\begin{array}{r}2,3 \\
0,5 \\
5,1 \\
2,8 \\
4,2 \\
6,0 \\
14,4 \\
5,6 \\
59,3\end{array}$ & $\begin{array}{c}5 \\
6 \\
17 \\
23 \\
32 \\
45 \\
76 \\
88 \\
216\end{array}$ & $\begin{array}{c}2,3 \\
2,8 \\
7,9 \\
10,6 \\
14,8 \\
20,8 \\
35,2 \\
40,7 \\
100,0\end{array}$ & $\begin{array}{c}7 \\
1 \\
13 \\
7 \\
9 \\
13 \\
33 \\
12 \\
128\end{array}$ & $\begin{array}{r}3,1 \\
0,4 \\
5,8 \\
3,1 \\
4,0 \\
5,8 \\
14,8 \\
5,4 \\
57,4\end{array}$ & $\begin{array}{c}7 \\
8 \\
21 \\
28 \\
37 \\
50 \\
83 \\
95 \\
223\end{array}$ & $\begin{array}{r}3,1 \\
3,6 \\
9,4 \\
12,6 \\
16,6 \\
22,4 \\
37,2 \\
42,6 \\
100,0\end{array}$ \\
\hline Total & 68 & 100,0 & & & 216 & 100,0 & & & 223 & 100,0 & & \\
\hline
\end{tabular}

\section{TABELA 10}

Aplicação da Escala 1 (Pré-natal), no ponto de corte 3, ao total de nascidos vivos em 1984 e aos óbitos de crianças menores de 1 ano nascidas em 1984 - Cotia e Vargem Grande Paulista.

\begin{tabular}{cccc}
\hline \multirow{2}{*}{ Escala } & \multicolumn{2}{c}{ Verdade } & Total \\
\cline { 2 - 3 } & $\begin{array}{c}\text { Risco } \\
\text { (Óbitos) }\end{array}$ & $\begin{array}{c}\text { Não Risco } \\
\text { (Não Óbitos) }\end{array}$ & \\
\hline Risco & 57 & 883 & 940 \\
Não Risco & 33 & 1.586 & 1.619 \\
\hline Total & 90 & 2.469 & 2.559 \\
\hline
\end{tabular}

ou seja, seleção de um grupo maior ou menor como de "alto risco." A Tabela 5 mostra os intervalos dos valores das RPCs para cada nível das escalas. As escalas para uso no pré-natal, parto e período neonatal apresentam os mesmos intervalos para cada grau da escala.

A Tabela 6 mostra a escala para o pré-natal com os oito graus em que foi dividida, correspondendo cada um deles a um intervalo para os valores da RPC. O nível zero (0) da escala corresponde aos indivíduos não pertencentes a nenhum grupo de risco e, portanto, com um total de pontos igual a 1 . O grau 1 da escala corresponde aos indivíduos cujo resultado final da multiplicação dos valores da RPC das 10 variáveis ficou em uma faixa entre 1,1 e 2,0. O grau 2 corresponde a um resultado final entre 2,0 e 2,5 e assim sucessivamente até o grau 8 e mais que corresponde ao intervalo 60,0 ou mais. Nota-se que $14,8 \%$ ( 22 casos) das mães do grupo de óbitos situaram-se no grau 8 ou mais da tabela, correspondendo a um total de pontos de 60 ou mais. Somente 2,8\% ( 6 casos) das mães do grupo de sobreviventes situou-se no mesmo grau da escala. Pode ser visto ainda que $42,3 \%$ ( 63 casos) das mães do grupo de óbitos apresentou um valor final dos seus pontos correspondente a 5 ou mais graus na escala de risco, enquanto que igual situação ocorreu somente com $9,7 \%$ (21 casos) das mães do grupo de sobreviventes.

Procedimento igual ao da escala de pré-natal foi feito para as 3 escalas seguintes, cada uma tendo oito níveis ou graus e correspondendo cada nível a um intervalo de valores para o resultado final da multiplicação das RPCs. As Tabelas 6, 7, 8 e 9 mostram a distribuição dos grupos de 6 bitos, sobreviventes e da amostra dos nascidos vivos em 1984 , nas quatro escalas.

Na sua aplicação, as escalas permitem uma definição dos grupos de risco e não risco, a partir da decisão sobre o ponto de corte nos seus níveis, que deverá discriminar em que grupo deverá ser incluído um indivíduo em particular. Assim, ainda na escala de pré-natal, se o nível 3 for o escolhido como ponto de corte, isto implicará que todas as mães cujo resultado final da multiplicação da razão dos seus produtos cruzados for igual ou superior a 2,5 serão consideradas de "risco" e todas aquelas cujo resultado for abaixo de 2,5 serão consideradas de "não risco". $\mathrm{Na}$ realidade, entre os indivíduos considerados de risco, há aqueles que não o são na verdade e denominam-se falsos positivos. Da mesma forma entre os indivíduos do grupo "não risco" há aqueles que de fato são de alto risco e, neste caso, são denominados de falsos negativos.

Aos diferentes níveis da escala corresponderam: um grupo de verdadeiros positivos (indivíduos do grupo de óbitos selecionados como de alto risco); um de verdadeiros negativos (indivíduos do grupo de sobreviventes selecionados como de baixo risco); um de falsos positivos (indivíduos do grupo de sobreviventes selecionados como de alto risco); e um de falsos negativos (indivíduos do grupo de óbitos selecionados como de baixo risco). 
As 94 mães (verdadeiros positivos) classificadas como de alto risco a partir do ponto de corte 3 (Tabela 6), na verdade, corresponderam a $63 \%$ do total de alto risco, ou seja, do total de 149 que pertenciam ao grupo de óbitos. Este valor, de fato, mede a sensibilidade do ponto de corte, ou seja, a sua capacidade de identificar os verdadeiros positivos. Por outro lado, este mesmo grau da escala definiu como de baixo risco 140 mães do grupo de sobreviventes (verdadeiros negativos), o que significou $65,8 \%$ do total de 216 mães do grupo de sobreviventes. Este valor de $65,8 \%$ corresponde a especificidade do ponto de corte, ou seja, da sua capacidade de identificar os verdadeiros negativos.

Estas características, acima descritas, das 4 escalas e dos seus pontos de corte, isto é, sensibilidade, especificidade, verdadeiros positivos, verdadeiros negativos, falsos positivos e falsos negativos são aspectos importantes a serem levados em conta, juntamente com a disponibilidade de recursos, no processo de implantação de uma estratégia de risco na redução da mortalidade infantil.

\section{5-COMENTÁRIOS}

O fato de se ter trabalhado em uma área delimitada, relativamente homogênea, é uma possível explicação para não ter sido encontrada associação entre algumas variáveis classicamente descritas como associadas à mortalidade infantil. Assim, por exemplo, a renda familiar per capita não mostrou associação. Ao que parece, há uma certa homogeneidade socio-econômica, na área, e alguns outros indicadores foram mais discriminatórios na identificação dos grupos de risco do que a renda.

As quatro escalas elaboradas como instrumento de identificaçăo dos grupos de alto risco tiveram como pressuposto sua utilização por profissionais não médicos; assim, elas não incluem informações médicas, isto é, dados de consulta ou exame laboratorial, e foram elaboradas a partir dos questionários respondidos pelas mães entrevistadas, e mesmo as questóes de morbidade dizem respeito à morbidade referida. Este fato torna possível a sua utilizaçăo a partir de uma entrevista com as mães clientes dos serviços de saúde. O melhor acompanhamento dos grupos selecionados, a partir das escalas, e contando então com informações clínicas e laboratoriais, poderá permitir melhor seleção, diferenciando, mesmo entre os grupos de risco, os indivíduos (gestantes e crianças) que, pela sua evolução clínica, mereçam cuidados específicos.

Estas escalas devem permitir tão somente a identificaçăo dos grupos que por serem de alto risco mereçam uma assistência especial. As características desta assistência deverăo ser definidas pelos programas de saúde. Algumas famflias necessitarão muito mais de orientação educativa e de suporte social do que de assistência médica ou tratamento clínico. Por outro lado, algumas mães necessitarão de um eficaz acompanhamento médico-laboratorial e provavelmente de acesso a tecnologias mais sofisticadas para superarem seus fatores de risco. $\mathrm{Na}$ verdade, o que vai causar impacto em nível populacional é a qualidade e eficiência dos programas de saúde; o trabalho inicial de seleção dos grupos nada mais é do que um primeiro passo, em um processo muito maior.

A análise da evolução dos indicadores de saúde e principalmente da saúde infantil nos municípios de Cotia e Vargem Grande Paulista parece indicar a necessidade de novas estratégias para manter a queda da mortalidade infantil. Houve nos últimos anos uma queda acentuada da mortalidade infantil e uma alteração significativa no seu padrão, com proporçőes semelhantes dos seus componentes neonatal e pós-neonatal. Esta última apresenta hoje valor relativamente baixo para a realidade do Estado e do País, devendo estar mais restrita aos chamados "bolsões" de pobreza, estando o seu controle na dependência de os serviços de saúde identificarem estes grupos e desenvolverem açóes adequadas às suas necessidades. A mortalidade neonatal também depende de uma estratégia igual, além de que seu controle, dependendo mais das ações de saúde do que do controle do meio ambiente, está na dependência de que os programas de saúde sejam capazes de facilitar o acesso dos grupos de alto risco à tecnologia adequada. Em ambas as circunstâncias, a disponibilidade de instrumentos preditivos deve ser de alguma utilidade.

Além disto, como foi descrito por Shapiro e col.12, os sobreviventes dos grupos de alto risco de óbito apresentam uma alta prevalência de morbidade, no primeiro ano de vida. Assim, ao selecionar-se um grupo de risco para acompanhamento especial, está sendo escolhido, de fato, um grupo com alto risco de adoecer, e a atenção privilegiada que esteja sendo dispensada ao grupo como um todo, na verdade, não deve somente reduzir um certo número de 6́bitos, mas também elevar o nível de saúde daquela população, que é justamente a mais necessitada.

Um exemplo de uso desta proposta pode ser visto aplicando-se a escala de pré-natal, com o ponto de corte 3, à amostra de nascidos vivos e ao grupo de óbitos. Pela Tabela 6, vê-se que este nível de corte selecionaria $63,1 \%$ das mães do grupo de 6 bitos como de alto risco e $36,8 \%$ da amostra de nascidos vivos em 1984 como, também, de alto risco.

O número de nascidos vivos em 1984 foi de 2.559 e o de óbitos de menores de um ano, em 1984 e 1985, foram, respectivamente, 94 e 85 . Destes 179 6bitos ocorridos nos dois anos seguidos, 90 foram de crianças nascidas em 1984, isto é, das 2.559 crianças nascidas em 1984, 90 morreram antes de completar um ano de idade.

O nível 3 da escala de pré-natal selecionaria 
assim (Tabela 10), 36,8\% das mães que deram à luz nascidos vivos em $1984(2.559 \times 0,368)$, ou seja, 940 como de alto risco e a diferença $(2.559$ - 940), 1.619, como de baixo risco. Da mesma forma este nível selecionaria $63,1 \%$ das mães que tiveram óbitos $(90$ $\times 0,631)$, isto é, 57 como de alto risco e $33(90-57)$ como de baixo risco. Assim, entre os 940 nascidos vivos de alto risco estariam incluídos 57 6́bitos, significando um coeficiente de mortalidade infantil igual $60,6 \%$ NV [(57/940) x 1000], e entre os 1.619 de baixo risco estariam incluídos 33 óbitos, traduzindo um coeficiente de mortalidade infantil de $20,4 \%$ NV [ $(33 / 1619) \times 1000]$.
A utilização do "enfoque de risco" teria o significado de, ao se trabalhar com estas duas populações, uma delas apresentando um risco de óbito infantil 3 vezes o da outra, estar priorizando-a em termos de assistência à saúde, sem contudo, o que é óbvio, deixar de fornecer uma assistência básica a população de menor risco.

A efetiva utilidade desses instrumentos, pela sua própria natureza, só poderá ser melhor avaliada após sua aplicação em um programa concreto de assistência materno-infantil, baseado no enfoque de risco.

CESAR, C.L.G. [Risk factors associated with infant mortality in two municipalities of the metropolitan area of S.Paulo (Brazil), 1984-1985. Proposal for a predictive scoring system. Rev. Saúde públ., S. Paulo, 24: 300-10, 1990.

ABSTRACT: The application of the concept of "risk" to maternal and child health was studied, the starting point being the World Health Organization's proposal for a "risk approach" in health services. The study was concerned with the development of a scoring system for the identification of groups at high risk of death during the first year of life, whether in the neonatal or the post-neonatal periods, and was based on a case-control study. The case group consistes of children under one year of life, whose deaths had been registered during the years 1984 and 1985 and whose parents lived in Cotia and Vargem Grande Paulista (149 cases). The control group was a probabilistic sample of 216 children born in 1984, which survived the first year of life and whose parents lived in the same area. All the mothers were interviewed by means of a questionnaire for the identification of independent variables associated with infant death. The statistically associated variables were grouped in four scales: to be used, respectively, in the antenatal period, prior to delivery and in the neonatal and post-neonatal periods. The variables were weighted in the scales according to the values of the odds ratio, and they have different cut points, each one having its own sensibility, specificity and predictive value.

KEYWORDS: Infant mortality. Risk factors. Risk groups. Predictive value of tests.

\section{REFERENCIAS BIBLIOGRÁFICAS}

1. BACHETT, E. M.; DAVIES, A. M.; PETROSBARVAZIAN, A. O enfoque de risco na assistência à saúde: com especial referência d̀ saúde materno-infantil, incluside planejamento familiar. Washington, D. C., Organização Panamericana da Saúde, 1985. (OPAS - Publicação Científica, 491).

2. BLACK, D.; MORRIS, S. N,; SMITH, C.; TOWNSEND, P. Inequalities in health; the Black report. New York, Penguin Books, 1982.

3. CESAR, C. L. G. Utilization of maternity and infant care in an urban district in Brazil. London, 1980. [M. Sc. Thesis - London School of Hygiene and Tropical Medicine].

4. GUIMARĀES, J. J. de L. \& FISCHMANN, A. Desigualdades na mortalidade infantil entre favelados e não-favelados no município de Porto Alegre, Rio Grande do Sul, em 1980. Bol. Ofic. sanit. panamer., 101: 19-38, 1986.
5. HART, J. T. The inverse care law. Lancet, 1: 405-12, 1971.

6. MCKEOWN, T. \& LOWE C. R. An introduction to social medicine. 2nd ed. London, Blackwell Scientific Publications, 1974.

7. MCKINLAY, J. B. Some approaches and problems in the study of the use of services: an overview. $J$. Hlth soc. Behav. 13: 115-52, 1972.

8. ORGANIZACION MUNDIAL DE LA SALUD. Metodo de atencion sanitaria de la madre y el niño basado en el concepto de riesgo. Ginebra, 1978. (OMS- Publicación en Offset, 39).

9. PAIM, J. S. \& COSTA, M. da C. As desigualdades na distribuição dos óbitos no município de Salvador -1980. Cad. saúde públ., Rio de Janeiro, 2: 312-33, 1986.

10. RICO-VELASCO, J. Marco de referencia para el estu- 
dio de la mortalidad infantil: politicas de salud y mortalidad infantil en las Américas; version preliminar. Washington, D. C., Organización Panamericana de la Salud, 1983. [Mimeografado].

11. SCHLESSELMAN, J. J. Case-control studies: design, conduct, analysis. Oxford, Oxford University Press, 1982.

12. SHAPIRO, S.; MACCORMICK, M. C.; STARFIELD, B. H.; KRISHER, J. P.; BROSS, D. Relevance of correlates of infant deaths for significant morbidity at 1 year of age. Amer. J. Obstet. Gynec., 136: 363-73, 1980.
13. WORLD HEALTH OORGANIZATION. A workbook on how to plan and carry out research on the risk approach in maternal and child health including family planning - Geneva, Division of Family Health, 1984. (Experimental Edition FHE/MCH/RA 84.1).

Recebido para publicação em 7/12/1989

Reapresentado em 26/3/1990

Aprovado para publicação em 28/3/1990 